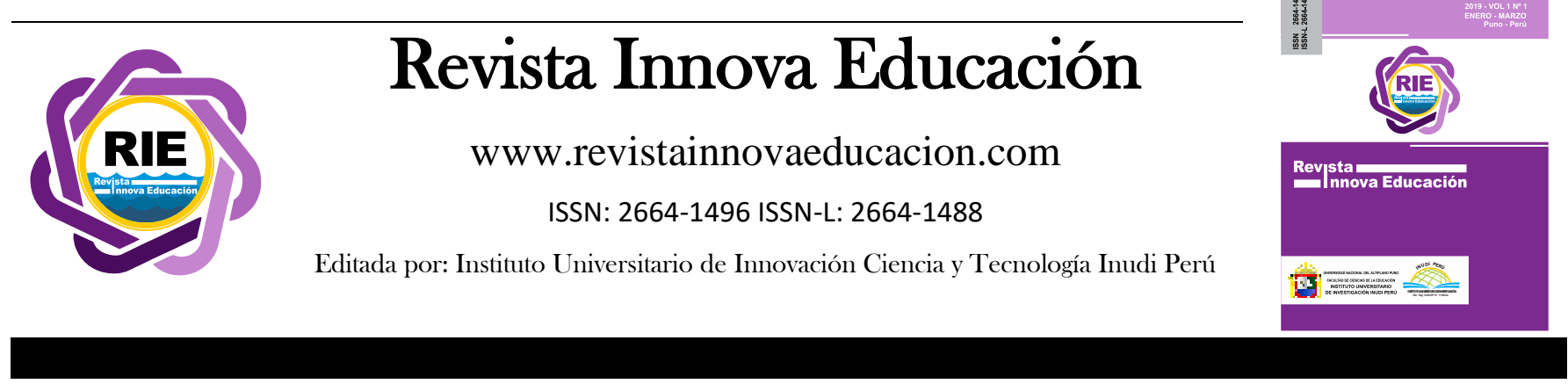

\title{
Hábitos de estudio: factor crucial para el buen rendimiento académico
}

\author{
Study habits: crucial factor for good academic environment \\ Wendy Soto \\ Universidad Nacional del Altiplano, Puno - Puno, Perú \\ https://orcid.org/0000-0003-1987-6400 \\ Nelly Rocha ${ }^{1}$ \\ Universidad Nacional del Altiplano, Puno - Puno, Perú \\ https://orcid.org/0000-0003-3418-5712
}

DOI: https://doi.org/10.35622/j.rie.2020.03.004

Recibido 25/06/2020/ Aceptado 25/07/2020 Publicado 25/07/2020

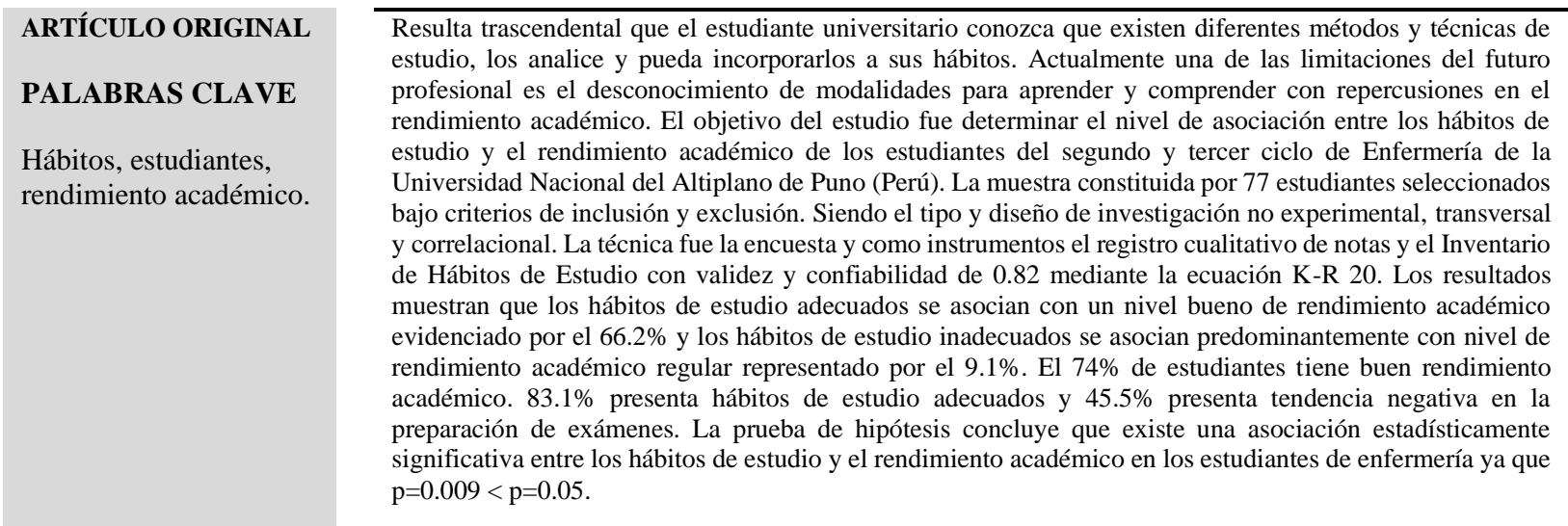

KEYWORDS

Habits, students, academic performance.

\begin{abstract}
It is important that university students recognize different methods and study techniques; and moreover, analyze and incorporate them into their habits. Currently, one of the limitations for the future academic development, is the lack of knowledge of learning and understanding modalities, whose impact affects the academic performance. The objective of the study was to determine the level of association between study habits and academic performance of students from the second and third cycle of the Faculty of Nursing at the National University of the Altiplano in Puno, Peru. The sample of the study was based on 77 students, selected under inclusion and exclusion criteria. The research has a non-experimental, transversal and correlational approach. The study technique was the survey with its instruments of qualitative note taking and the Inventory of Study Habits, whose validity and reliability has a value of 0.82 through the equation K$\mathrm{R} 20$. The results show that study habits are associated with a good level of academic performance, proven by $66.2 \%$ of students; on the other hand, the use of inadequate study habits are predominantly associated with an average academic performance represented by $9.1 \%$ of students. Also, $74 \%$ of students have good academic performance, $83.1 \%$ have adequate study habits and $45.5 \%$ have a negative trend during test
\end{abstract}

\footnotetext{
${ }^{1}$ Correspondencia: nmrocha@unap.edu.pe
} 


\section{INTRODUCCIÓN}

Edel (2003) identifica al rendimiento académico como un constructo capaz de adoptar valores cuantitativos y cualitativos a través de los cuales existe una aproximación a la evidencia del perfil de conocimientos, habilidades, valores y actitudes desarrollados por el estudiante en el proceso de enseñanza-aprendizaje. Por otro lado, los hábitos de estudio se definen como métodos y técnicas de estudio que los estudiantes aprenden y desarrollan de manera rutinaria en relación al proceso de enseñanza-aprendizaje, pudiendo ser adecuados o inadecuados para el rendimiento académico (Eduardo \& Garza, 2012).

Diversas investigaciones concluyen que los estudiantes universitarios, entre ellos estudiantes de enfermería, evidencian bajo rendimiento académico (Mercado et al., 2019; Rosell, 2015). La QS World University Rankings (2019) señala que solo 93 universidades latinoamericanas fueron nombradas entre las mejores mil del mundo (Rankings, 2018), y según el ranking desarrollado por la revista Times Higher Education que mide el desempeño de las universidades basándose en indicadores relacionados a la calidad de la enseñanza, investigación, transferencia de conocimiento y la internacionalización de la universidad, únicamente la Pontificia Universidad Católica del Perú se encuentra dentro del top 50 a nivel de América Latina (SUNEDU, 2019).

Un estudio de la Organización para la Cooperación y el Desarrollo Económico (OCDE) basado en datos de los 64 países participantes en el Programa para la Evaluación Internacional de los Alumnos (PISA) señala que Latinoamérica está por debajo de los estándares globales de rendimiento académico y puntualiza que Perú, Colombia, Brasil y Argentina se encuentran entre las diez naciones cuyos estudiantes tienen el rendimiento más bajo en áreas como matemática, ciencia y lectura (BBC, 2019).

A nivel nacional el Instituto Nacional de Estadística e Informática (2018) reporta que el 93,2\% de la población de 15 a 29 años que asistió en el 2017 a educación superior universitaria, aprobó y pasó de ciclo. Los departamentos con los porcentajes más altos son la Provincia Constitucional del Callao (98,5\%), Cajamarca (98,3\%), Provincia de Lima y Ayacucho (96,7\% en cada caso), Ucayali (95,7\%), San Martín (95,2\%), Moquegua (94,5\%), entre otros y los departamentos con menores porcentajes registrados son Puno (91.3\%), Madre de Dios (85.7\%), Amazonas (77,3\%) y Junín (77,7\%). 
En el ámbito local, un estudio concerniente al rendimiento académico en universitarios de la Escuela Profesional de Enfermería, reporta que 52\% de estudiantes evidencia rendimiento académico regular con notas que oscilan entre 11 y 13 (Uturunco, 2016). Asimismo, el Vicerrectorado Académico informa que en el segundo semestre académico 2019 se registraron 179 estudiantes con cuarta matrícula, 1016 suspendidos, 316 fueron retirados y 34 abandonaron sus estudios, y específicamente en la Facultad de Enfermería se identificó 2 estudiantes con cuarta matrícula, 16 suspendidos, 4 retirados y 3 que abandonaron la carrera profesional (Universidad Nacional del Altiplano, 2019).

El bajo rendimiento académico puede generar fracaso académico considerado un problema multicausal que afrontan los estudiantes y que los lleva en poco tiempo a desertar o ser expulsados del sistema educativo (Contreras et al., 2008). Barbieri (2016) confirma que el bajo rendimiento académico es un factor determinante en el proceso de deserción, lo que se traduce en un bajo nivel educativo en la población, perjudicando el capital humano y educativo necesario para que la población se inserte en empleos remunerados que les ayude a salir de la situación de pobreza.

Al entrevistar a los estudiantes de los primeros ciclos de la Facultad de Enfermería sobre sus hábitos de estudio manifestaron que dejan las tareas para último momento, no profundizan los temas aprendidos, estudian un día u horas antes del examen, elaboran plagios y durante el desarrollo de clases tienen poca disponibilidad para prestar atención a la información de los docentes; lo que evidentemente tiene repercusiones en el rendimiento académico y así lo demuestra el cuadro de méritos de estudiantes del I y II ciclo 2019-I donde el promedio ponderado oscila entre 13 y 14 y el 17\% tiene cursos desaprobados (Universidad Nacional del Altiplano, 2019).

Entre los factores que podrían determinar el bajo rendimiento académico de los universitarios destacan la competencia cognitiva, motivación, condición cognitiva (falta de hábitos de estudio o técnicas de trabajo intelectual, estilos de aprendizaje inadecuados), el auto concepto académico, autoeficacia percibida, asistencia a clases, inteligencia, formación académica previa a la universidad, nota de acceso, niveles de conocimiento no adecuados a las exigencias universitarias, desarrollo inadecuado de aptitudes específicas acordes con el tipo de carrera elegida y aspectos de índole actitudinal (Garbanzo, 2007). Desde la perspectiva de los docentes las variables que más inciden en el bajo rendimiento académico son el escaso nivel de conocimientos previos, falta de autocontrol, auto exigencia y responsabilidad (Tejedor \& García, 2007). Destacan también el deficiente aprovechamiento de las horas de tutoría, la baja estimulación institucional para las tareas docentes, el insuficiente dominio de técnicas de estudio y su falta de esfuerzo para centrarse en el estudio (Meraz et al., 2013). 
La aplicación de hábitos de estudio es imprescindible para todos los alumnos pues la falta de uso de estas herramientas imposibilita el aprendizaje óptimo (Bedolla, 2018). Lara (2016) concluye que los estudiantes no poseen buenos hábitos de estudio, no saben leer comprensivamente, no utilizan el subrayado y no evidencian buena asimilación de los contenidos.

Si bien es cierto existen estudios previos concernientes a los hábitos de estudio y rendimiento académico en universitarios de las distintas escuelas profesionales tanto a nivel internacional y nacional, son escasos en nuestra región y no se evidencian estudios concernientes al tema hábitos de estudio en el área de enfermería, lo que motivó esta investigación para responder a la interrogante ¿Existe asociación entre los hábitos de estudio y el rendimiento académico de los estudiantes de enfermería de la Universidad Nacional del Altiplano, Puno-2019?

\section{MÉTODO Y MATERIALES}

\section{Objetivos}

- Determinar el nivel de asociación entre los hábitos de estudio y el rendimiento académico de los estudiantes del segundo y tercer ciclo de la Facultad de Enfermería de la Universidad Nacional del Altiplano de Puno (Perú).

- Identificar el nivel de rendimiento académico de los estudiantes del segundo y tercer ciclo de la Facultad de Enfermería.

- Identificar los hábitos de estudio en función del valor final: adecuados e inadecuados.

- Identificar los hábitos de estudio en las dimensiones: forma de estudio, resolución de tareas, preparación para exámenes, forma de escuchar la clase y acompañamiento al estudiar.

\section{Tipo y diseño}

El estudio fue de tipo correlacional con diseño no experimental y de corte transversal.

\section{Muestra}

El muestreo fue probabilístico estratificado constituido por 77 estudiantes del segundo y tercer ciclo de la Facultad de Enfermería correspondiente al año académico 2019.

\section{Técnicas e instrumentos}

La técnica aplicada fue la encuesta y como instrumentos de recolección de datos, el registro cualitativo de notas y el Inventario de Hábitos de estudio CASM-85 revisión 2014 (Vicuña, 1999). 
El instrumento cuenta con validez obtenida mediante el análisis ítem-test corregido el cual mostró índices de homogeneidad que se hallan entre los niveles de bueno a elevado. Así mismo, el autor desarrolló el análisis factorial confirmatorio empleando el método de máxima verosimilitud evidenciando un ajuste aceptable con el modelo teórico y también el análisis factorial exploratorio el cual indica una muestra adecuada de ítems. La confiabilidad reportó un valor de 0.827 mediante el estadígrafo KR20.

\section{Procesamiento estadístico de datos}

Tras la verificación y organización de los datos obtenidos estos se procesaron en el programa SPSS versión 24.

Para identificar la asociación entre los hábitos de estudio y el rendimiento académico y por tratarse de variables cualitativas se utilizó la prueba estadística ji-cuadrado $\left(\mathrm{X}^{2}\right)$ teniendo como regla de decisión:

Si $\mathrm{X}_{\mathrm{c}}^{2}<\mathrm{X}_{\mathrm{t}}^{2}$ No se rechaza la hipótesis nula.

Si $p>0.05$ No se rechaza la hipótesis nula.

\section{Consideraciones éticas}

Antes de la recolección de datos, se aplicó el proceso del consentimiento informado siguiendo las pautas de la Declaración de Helsinki, Código de Nuremberg y Normas CIOMS, dando una información verbal y escrita del protocolo de investigación y solicitando el consentimiento informado escrito.

\section{RESULTADOS}

Tabla 1. Hábitos de estudio y rendimiento académico en estudiantes de enfermería de la UNA Puno (Perú 2019).

\begin{tabular}{ccccccccc}
\hline Hábitos & \multicolumn{1}{c}{ Nivel de Rendimiento Académico } \\
\hline & \multicolumn{2}{c}{ Regular } & \multicolumn{2}{c}{ Bueno } & \multicolumn{2}{c}{ Excelente } & \multicolumn{2}{c}{ Total } \\
& fi & $\%$ & fi & $\%$ & fi & $\%$ & fi & $\%$ \\
Hábitos & 7 & 9.1 & 6 & 7.8 & 0 & 0.0 & 13 & 16.9 \\
$\begin{array}{c}\text { Inadecuados } \\
\text { Hábitos }\end{array}$ & 10 & 13.0 & 51 & 66.2 & 3 & 3.9 & 64 & 83.1 \\
$\begin{array}{c}\text { Adecuados } \\
\text { Total }\end{array}$ & 17 & 22.1 & 57 & 74.0 & 3 & 3.9 & 77 & 100.0 \\
\hline
\end{tabular}

Fuente: Inventario de Hábitos de Estudio CASM-85 y Registro cualitativo de promedio ponderado semestral. 
Los hábitos de estudio adecuados se relacionan con rendimiento académico bueno evidenciado por el $66.2 \%$, seguido del $13 \%$ que muestra nivel de rendimiento regular y solo el $3.9 \%$ nivel excelente. Por otro lado, los hábitos de estudio inadecuados se relacionan predominantemente con nivel de rendimiento académico regular (9.1\%), el 7.8\% de estudiantes presenta un nivel bueno.

Al aplicar la prueba estadística ji-cuadrado se obtuvo lo siguiente:

\section{Pruebas de chi-cuadrado}

\begin{tabular}{lr|r|r}
\hline & Valor & \multicolumn{1}{c}{ df } & \multicolumn{1}{c}{ Valor $p$} \\
\hline ji-cuadrado de Pearson & 9,400 & 2 &, 009 \\
\hline Razón de verosimilitud & 8,525 & 2 &, 014 \\
\hline Asociación lineal por lineal & 8,662 & 1 &, 003 \\
\hline N de casos válidos & 77 & & \\
\hline
\end{tabular}

$X_{c}^{2}=9.4>X_{t}^{2}=5.9$ por consiguiente se rechaza la Ho "No existe asociación entre los hábitos de estudio y el rendimiento académico en estudiantes de Enfermería de la Universidad Nacional del Altiplano, Puno-2019". Asimismo, en la significación asintótica bilateral se obtuvo P=0.009, siendo este un valor menor que $\mathrm{P}=0.05$, por tanto, existe asociación estadísticamente significativa entre los hábitos de estudio y el rendimiento académico.

Tabla 2. Nivel de rendimiento académico en estudiantes de enfermería de la UNA Puno (Perú 2019).

\begin{tabular}{ccc}
\hline Rendimiento & Frecuencia & $\%$ \\
\hline Regular & 17 & 22.1 \\
Bueno & 57 & 74.0 \\
Excelente & 3 & 3.9 \\
Total & 77 & 100.0 \\
\hline
\end{tabular}

Fuente: Registro de promedio cualitativo ponderado semestral

Se observa que el $74 \%$ de estudiantes evidencia buen nivel de rendimiento académico, seguido del $22.1 \%$ con nivel regular, $3.9 \%$ que presenta nivel excelente. 
Tabla 3. Hábitos de estudio en estudiantes de enfermería de la UNA Puno (Perú 2019)

\begin{tabular}{ccccc}
\hline Niveles & Categorías & $\mathbf{f i}$ & $\%$ & Total \\
\hline Hábitos & Muy & 0 & 0 & \\
Inadecuados & $\begin{array}{c}\text { Negativo } \\
\text { Negativo }\end{array}$ & 0 & 0 & \\
& $\begin{array}{c}\text { Tendencia } \\
\text { Negativa }\end{array}$ & 13 & $16.9 \%$ & \\
& Tendencia & 31 & 40.3 & \\
Hábitos & Positiva & & & $83.1 \%$ \\
Adecuados & Positiva & 27 & 35.1 & \\
& Muy Positivo & 6 & 7.8 & \\
& Total & 77 & 100 & $100 \%$ \\
\hline
\end{tabular}

Fuente: Inventario de Hábitos de Estudio CASM-85 Revisión 2014

El $83.1 \%$ de estudiantes presenta hábitos de estudio adecuados, mientras que $16.9 \%$, inadecuados. En otra instancia, el $40.3 \%$ tiene tendencia positiva y $16.9 \%$ posee tendencia negativa.

Tabla 4. Dimensiones de los hábitos de estudio de los estudiantes de enfermería de la UNA. Puno (Perú 2019)

\begin{tabular}{|c|c|c|c|c|c|c|c|c|c|c|c|}
\hline \multirow{3}{*}{ Niveles } & \multicolumn{11}{|c|}{ Dimensiones de los hábitos de estudio } \\
\hline & \multirow[t]{2}{*}{ Categorías } & \multicolumn{2}{|c|}{$\begin{array}{l}\text { Forma de } \\
\text { Estudio }\end{array}$} & \multicolumn{2}{|c|}{$\begin{array}{l}\text { Resolución } \\
\text { de Tareas }\end{array}$} & \multicolumn{2}{|c|}{$\begin{array}{c}\text { Preparación } \\
\text { para } \\
\text { Exámenes }\end{array}$} & \multicolumn{2}{|c|}{$\begin{array}{c}\text { Forma de } \\
\text { escuchar la } \\
\text { clase }\end{array}$} & \multicolumn{2}{|c|}{$\begin{array}{c}\text { Acompañamiento } \\
\text { al estudiar }\end{array}$} \\
\hline & & fi & $\%$ & fi & $\%$ & fi & $\%$ & fi & $\%$ & fi & $\%$ \\
\hline \multirow{5}{*}{$\begin{array}{c}\text { Hábitos } \\
\text { Inadecuados }\end{array}$} & Muy Negativo & 0 & 0 & 0 & 0 & 0 & 0 & 0 & 0 & 0 & 0 \\
\hline & Negativo & 0 & 0 & 2 & 2.6 & 6 & 7.8 & 2 & 2.6 & 3 & 3.9 \\
\hline & Tendencia & & & & & & & & & & \\
\hline & Negativa & 7 & 9.1 & 21 & 27.3 & 35 & 45.5 & 9 & 11.7 & 13 & 16.9 \\
\hline & Tendencia & & & & & & & & & & \\
\hline Hábitos & Positiva & 31 & 40.3 & 33 & 42.9 & 23 & 29.9 & 17 & 22.1 & 16 & 20.8 \\
\hline \multirow[t]{3}{*}{ Adecuados } & Positivo & 29 & 37.7 & 16 & 20.8 & 13 & 16.9 & 31 & 40.3 & 23 & 29.9 \\
\hline & Muy Positivo & 10 & 13 & 5 & 6.5 & 0 & 0 & 18 & 23.4 & 22 & 28.6 \\
\hline & Total & 77 & 100 & 77 & 100 & 77 & 100 & 77 & 100 & 77 & 100 \\
\hline
\end{tabular}

Fuente: Inventario de Hábitos de Estudio CASM-85 Revisión 2014

De las dimensiones que evalúan el inventario de hábitos de estudio destaca la relación entre la forma de escuchar la clase (40.3\%) y hábitos adecuados positivos, luego el acompañamiento al estudiar con nivel positivo (29.9\%). Las dimensiones resolución de tareas y forma de estudio presentan tendencia positiva con $42.9 \%$ y $40.3 \%$ respectivamente, y el área preparación para exámenes presenta tendencia negativa $(45.5 \%)$. 


\section{DISCUSIÓN}

Los resultados evidencian que existe asociación entre los hábitos de estudio y el rendimiento académico de los estudiantes de enfermería, porque el análisis estadístico muestra un valor para ji calculada de 9.4 mayor que la ji de tablas que es 5.9. Se obtuvo un valor $p=0.009$ menor que $\mathrm{p}=0.05$, por tanto, se rechaza la hipótesis nula.

A efecto de desarrollar un análisis más exhaustivo se aplicó la prueba estadística a cada dimensión del inventario de hábitos de estudio en asociación con el rendimiento académico teniendo como resultado que cuatro de las cinco dimensiones comprendidas en el inventario (forma de estudio, preparación para exámenes, forma de escuchar la clase, acompañamiento al estudiar) evidencian valor de ji-cuadrado calculada mayor que la tabulada y significación asintótica menor a 0.05. (Las dimensiones forma de estudio y preparación para exámenes poseen 0.008 de significación; la dimensión forma de escuchar la clase, 0.030 y la dimensión acompañamiento al estudiar 0.022) indicando la asociación entre las dimensiones de hábitos de estudio y el rendimiento académico. Este resultado se asemeja al obtenido por Dominguez (2018) identificando que las dimensiones: forma de estudio, preparación para exámenes y forma de escuchar la clase se asocian con el rendimiento académico.

Los resultados obtenidos en el presente estudio son similares a los hallazgos reportados por Cruz \& Quiñones (2011), Gloriosa et al.,(2016), Jurado (2018), Gonzáles (2015), Grados \& Alfaro (2015), Llacsa (2010) y Vásquez (2018) que evidencian la asociación entre los hábitos de estudio y el rendimiento académico, donde los estudiantes que tienen adecuados hábitos de estudio poseen niveles altos de rendimiento académico y los que tienen inadecuados hábitos de estudio, bajo rendimiento académico. La semejanza de hallazgos permite afirmar la influencia crucial que ejercen los hábitos de estudio en el rendimiento constituyéndose en un aspecto determinante en el ámbito de la educación superior.

Durante el periodo de formación profesional de los estudiantes es imprescindible tener buen rendimiento académico ello implica la conjunción de una serie de factores personales, sociales e institucionales, siendo principalmente relevante los aspectos inherentes al estudiante como los hábitos de estudio (Garbanzo, 2007; Tejedor \& García, 2007). Torres et al., (2009) indican que el hábito de estudio representa en la vida académica un factor preponderante para alcanzar el éxito académico. 
Resulta trascendental que el estudiante universitario conozca que existen diferentes métodos y técnicas de estudio, los analice y pueda incorporarlos a sus hábitos. Actualmente una de las limitaciones del futuro profesional es el desconocimiento de modalidades para aprender y comprender porque no tienen un método racionalizado, un esquema mental que organice y dirija la acción de estudiar hacia el objetivo de la comprensión, Hernández (1996). El buen rendimiento no solo depende de la inteligencia y el esfuerzo sino también de la eficacia de los métodos y técnicas de estudio, Andrade et al., (2018).

El rendimiento académico tiene diferentes niveles cada uno con características específicas que desarrolla el estudiante. En el nivel excelente evidencia el logro de los aprendizajes esperados demostrando un manejo responsable y muy satisfactorio en todas las tareas propuestas; en el nivel bueno, demuestra el logro de los aprendizajes previstos en el tiempo proyectado; el rendimiento académico regular significa que el estudiante se encuentra en proceso de lograr los aprendizajes esperados para lo que requiere acompañamiento durante un tiempo razonable (Jurado, 2018).

Touron (1984) expresa que el rendimiento se constituye en la calificación numérica y cualitativa que si es sólida será el reflejo de un aprendizaje o del logro de objetivos establecidos con anterioridad, permite establecer en qué medida los estudiantes han logrado cumplir los objetivos educativos tanto en aspectos cognoscitivos y otros relacionados además de permitir obtener información para establecer estándares. Taba (1974) señala que los registros del rendimiento académico son básicamente esenciales para el diagnóstico de hábitos de estudio y habilidades, aspectos que pueden ser analizados como proceso concluyente del nivel y resultado final. El rendimiento académico es producto del esfuerzo y la capacidad de trabajo que posee el estudiante, el conocer estas variables conducirá a un análisis más concienzudo del éxito o fracaso académico (Ortega, 2012).

Los resultados obtenidos en el presente estudio se asemejan a los hallazgos referidos por Cruz \& Quiñones (2011) en cuyo estudio realizado en estudiantes de enfermería identificó que gran porcentaje presenta rendimiento académico entre bueno y regular, sin embargo, difiere con los hallazgos obtenidos por Uturunco (2016), Grados \& Alfaro (2015), LLacsa (2010), Dominguez (2018) y Vásquez (2018) en los que se destaca el rendimiento académico regular, ello podría deberse a la diferencia de criterios de evaluación del rendimiento académico establecido en cada universidad, como destaca Garbanzo (2007) cada universidad determina criterios evaluativos propios para obtener un promedio ponderado de las materias que cursa el estudiante donde se consideran elementos como la cantidad de materias, el número de créditos y el valor obtenido en cada una de ellas y los hábitos de estudio influirían considerablemente. 
En el estudio los resultados sobre hábitos de estudio según niveles y categorías muestran que gran porcentaje de los estudiantes posee hábitos de estudio adecuados situándose predominantemente en la tendencia positiva; sin embargo, poseen técnicas de estudio y/o formas de recoger información aprendidas que dificultan su aprendizaje óptimo. Estos resultados son semejantes a los hallazgos de Jurado (2018), Grados \& Alfaro (2015) y Dominguez (2018) pero difieren de Cruz \& Quiñones (2011), LLacsa (2010), Vásquez (2018) porque los estudiantes evidencian principalmente hábitos inadecuados. La diferencia de resultados podría deberse a que emplearon instrumentos como el cuestionario de Gilbert Wrenn y adaptaciones del Inventario CASM-85, a la variación sobre los modos de evaluación empleados por los docentes en cada universidad, desconocimiento de hábitos de estudio y a la formación académica recibida en el nivel secundario.

Es importante que el estudiante universitario conozca los diversos métodos y técnicas de estudio, los analice e incorpore a sus hábitos, en esta tarea resulta fundamental la opinión y guía del profesor debido a que los métodos de estudio no poseen una sola forma de aplicarse, no son rígidos, representan para el estudiante una manera de aprender haciendo mayor énfasis en los procesos mentales que debe desarrollar (Cruz \& Quiñones, 2011) de esta manera el estudiante irá desarrollando métodos y técnicas de estudio que en relación al proceso interactivo de la enseñanza aprendizaje se van consolidando en formas habituales de estudiar.

Los especialistas en didáctica que en sus evaluaciones plantean preguntas de análisis donde la reflexión es fundamental, descubren que sus estudiantes fracasan atribuyéndolo a sus limitaciones sin identificar que solo están respondiendo a la manera como fueron instruidos (Vicuña, 1999).

Actualmente algunos estudiantes ingresan a la universidad carentes de orientación, indudablemente esta falla involucra a las formas de estudio que han de seguir, si el estudiante no cuenta con una base sólida de hábitos de estudio esta carencia impactará negativamente en las actividades que lleve a cabo en su formación académica y profesional (Mondragón et al., 2017).

Los resultados encontrados sobre los hábitos de estudio en las dimensiones forma de escuchar la clase y acompañamiento al estudiar, presentan principalmente nivel positivo, en forma de estudio y resolución de tareas predomina la tendencia positiva, pero en la dimensión preparación para exámenes hay tendencia negativa y requieren ser reforzados. Este resultado es similar al obtenido por Dominguez (2018). Covey (2013) recomienda que el estudiante debe repasar lo realizado en clases tan pronto como pueda después de haber concluido sus clases, ordenar sus 
notas y revisarlas periódicamente, el repaso constante es de suma importancia para que pueda retener, asimilar e interiorizar todo lo aprendido.

Resulta necesario diferenciar memorización y comprensión, la primera es una actividad de horizontes limitados, en tanto que la comprensión es más un estudio dinámico de horizontes abiertos que impulsa al cambio (F. Vásquez, 2010). La comprensión requiere de ayudas prácticas para materializarse siendo estas las técnicas de estudio que el estudiante puede distinguir y poner en práctica tales como el subrayado, la lectura, uso del diccionario, elaboración de resúmenes entre otros (Tierno, 2012).

En ocasiones el estudiante pese a haber estudiado en el último momento puede llegar a obtener buena nota, sin embargo, si se quiere realmente lograr el dominio de la asignatura o desarrollar una mente culta es imprescindible esforzarse honestamente día a día por aprender, desarrollar un proceso de aprendizaje continuo y dinámico (Covey, 2013).

El uso de técnicas adecuadas para la resolución de tareas permite al estudiante realizar de manera más sencilla sus actividades académicas, una de ellas es la organización del tiempo (E. Gonzáles, 2018). La organización del tiempo implica adaptarse al trabajo que realiza y guiarse de un horario establecido (Ramon, 2012). Una adecuada administración del tiempo hace posible distribuir equilibradamente la jornada diaria y semanal, facilita la concentración al crear el hábito de estudiar determinadas materias en un instante y lugar determinado permitiendo aprovechar el tiempo libre para recreación, actividades de ocio o descanso (E. Gonzáles, 2018).

La educación es sostenible cuando es de calidad y se hace sostenible si en el proceso de adquisición de aprendizajes se aplicaron las técnicas de estudio y se formaron hábitos de estudio adecuados (Bedolla, 2018). Si se planifica la actividad intelectual dentro de la universidad el rendimiento académico se incrementará y la probabilidad de culminar satisfactoriamente los estudios universitarios será alta (Ramon, 2012).

\section{CONCLUSIONES}

Queda demostrado que existe asociación estadísticamente significativa entre los hábitos de estudio y el rendimiento académico de los estudiantes de enfermería de segundo y tercer ciclo de la Universidad Nacional del Altiplano Puno (Perú) a través de la prueba estadística ji-cuadrado cuyos resultados evidencian una significación asintótica de 0.009. Las dimensiones que principalmente se asocian con el rendimiento académico son: forma de estudio, preparación de exámenes, forma de escuchar la clase y el acompañamiento al estudiar. La dimensión preparación de exámenes posee tendencia negativa. 
Para superar las brechas encontradas, se hace necesario que al inicio de la carrera de enfermería se trabaje con los estudiantes un conjunto de buenos hábitos de aprendizaje y de estudio a fin de lograr resultados más homogéneos al final de la formación profesional, teniendo en cuenta que los estudiantes de enfermería proceden de diferentes tipos de instituciones educativas secundarias diseminadas en toda la región Puno y otras regiones vecinas trayendo consigo diferentes prácticas y experiencias culturales.

\section{REFERENCIAS BIBLIOGRÁFICAS}

Andrade, I., Facio, S., Quiroz, A., Alemán, L., Flores, M., \& Rosales, M. (2018). Atitude, hábitos de estudo e rendimento académico: Abordagem desde a teoria da ação racional. Enfermería Universitaria, 15(4). https://doi.org/10.22201/eneo.23958421e.2018.4.533

Barbieri, J. G. J. (2016). Rendimiento académico como determinante de la deserción en la Escuela de Economía de la Universidad Católica Andrés Bello.

BBC. (2019, junio). BBC News Mundo.

Bedolla, R. (2018). Programa educativo enfocado a las técnicas y hábitos de estudio para lograr aprendizajes sustentables en estudiantes de nuevo ingreso al nivel superior. Revista Iberoamericana de Educación, 76(2), 73-94. https://doi.org/10.35362/rie7622959

Contreras, K., Caballero, C., Palacio, J., \& Pérez, A. M. (2008). Factores asociados al fracaso académico en estudiantes universitarios de Barranquilla (Colombia). Psicol. Caribe, 22, 110135.

Covey, S. (2013). Los 7 hábitos de la gente altamente efectiva. (Paidós (ed.); p. 373).

Cruz, F., \& Quiñones, A. (2011). Hábitos de estudio y rendimiento académico en enfermería, Poza Rica, Veracruz México. Actualidades Investigativas en Educación, 11(3). https://doi.org/10.15517/aie.v11i3.10219

Dominguez, C. (2018). Hábitos de estudio y rendimiento académico de la asignatura de Biología en los estudiantes del II ciclo de Medicina en una Universidad Particular de Trujillo, 2016. César Vallejo.

Edel, R. (2003). El rendimiento académico: concepto, investigación y desarrollo. REICE. Revista Iberoamericana sobre calidad, Eficacia y Cambio en Educación, 1, 0.

Eduardo, Á., \& Garza, V. (2012). Los hábitos de estudio y motivación para el aprendizaje de los alumnos en tres carreras de ingeniería. Revista de la Educación Superior, XLI (3)(163), 6787. 
Garbanzo, G. (2007). Factores asociados al rendimiento académico en estudiantes universitarios, una reflexión desde la calidad de la Educación Superior Pública. Educación, 31.

Gloriosa, M., Vegas, P. De, Rosario, Z., Acosta, S., Oriente, P., Aquino, R., López, L., Arévalo, I., \& Daza, J. (2016). Hábitos de estudio y rendimiento académico en estudiantes de enfermería de la Universidad Peruana del Oriente. 6(2), 179-204.

Gonzáles, C. (2015). Hábitos de estudio y rendimiento académico de los estudiantes de Fundamentos de Enfermería, 2014.

Gonzáles, E. (2018). Hábitos de estudios y rendimiento académico en estudiantes del primer semestre de la Facultad de Educación de la Universidad Nacional Mayor de San Marcos2015. 193.

Grados, J., \& Alfaro, R. (2015). Hábitos de estudio y rendimiento académico en estudiantes del 1. ${ }^{\circ}$ año de Psicología de la Universidad Peruana Unión, Lima, Perú. Revista Científica de Ciencias de la Salud, 6(2), 48-53. https://doi.org/10.17162/rccs.v6i2.203

Hernández, F. (1996). Metodología del Estudio: Como estudiar con rapidez (McGraw-Hill (ed.)).

INEI. (2018). Perú: Indicadores de educación por departamento del 2007 al 2017. 99-107.

Jurado, N. (2018). Hábitos de estudios y rendimiento académico en estudiantes de Medicina Humana de la Universidad San Pedro - Chimbote, 2017. 1-60.

Lara, R. (2016). Fortalecimiento de los hábitos de estudios de los estudiantes Repitentes del grado $7^{\circ} \mathrm{A}$ en la Institución Educativa Rafael Núñez Sede Principal del Municipio de SincelejoSucre. En Corporación Universitaria del Caribe. https://doi.org/10.1017/CBO9781107415324.004

LLacsa, J. (2010). Los hábitos de estudio, Autoestima y su relación con el rendimiento académico de los estudiantes de la Facultad de Enfermería de la Universidad Nacional Jorge Basadre Grohmann. Jorge Basadre Grohmann.

Meraz, B., García, M., Candil, A., \& García, R. (2013). Asesoría académica: un recurso para los estudiantes que presentan el examen extraordinario de microbiología y parasitología. Investigación en Educación Médica, 2(7), 122-128. https://doi.org/10.1016/s20075057(13)72700-5

Mercado, C., Illesca, M., \& Hernández, A. (2019). Relación entre estrategias de aprendizaje y rendimiento académico: estudiantes de enfermería, Universidad Santo Tomás. Enfermería Universitaria. 
Mondragón, C., Cardoso, D., \& Bobadilla, S. (2017). Hábitos de estudio y rendimiento académico. Caso estudiantes de la licenciatura en Administración de la Unidad Académica Profesional Tejupilco, 2016 / Study habits and academic performance: A research study of Business Administration undergraduate student. RIDE Revista Iberoamericana para la Investigación y el Desarrollo Educativo, 8(15), 661-685. https://doi.org/10.23913/ride.v8i15.315

Ortega, V. (2012). Hábitos de estudio y Rendimiento Académico en estudiantes de segundo de secundaria en una Institución Educativa del Callao. Universidad San Ignacio de Loyola.

Ramon, J. (2012). Técnicas de Estudio. En Dirección de Educación a Distancia de la Universidad Alas Peruanas (p. 120). http://josemramon.com.ar/wp-content/uploads/2-Estudiar-y-escribiren-la-Universidad-Tecnicas-de-estudio-para-universitarios.pdf

Rankings, Q. W. U. (2018). QS World University Rankings 2018. QS Quacquarelli Symonds.

Rosell, M. (2015). Carga laboral y rendimiento académico de los estudiantes de enfermería universidad nacional Toribio Rodríguez de Mendoza, Chachapoyas 2014. Universidad Nacional Toribio Rodríguez de Mendoza de Amazonas. Tesis para optar por el título de licenciada de Enfermería., 60. https://doi.org/10.1016/j.mfglet.2018.06.003

SUNEDU. (2019). Informe Bienal sobre la Realidad Universitaria. Informe Bienal sobre la Realidad Universitaria.

Taba, H. (1974). Elaboración del Curriculo. Elaboración del Currículum, 1-14.

Tejedor, F., \& García, A. (2007). Causas del bajo rendimiento del estudiante universitario (en opinión de los profesores y alumnos): propuestas de mejora en el marco del EEES. Revista de educación, 342, 419-442.

Tierno, B. (2012). Las mejores técnicas de estudio. Ediciones Martínez Roca.

Torres, M., Tolosa, I., Urrea, M. del C., \& Monsalve, A. (2009). Hábitos de estudio vs fracaso escolar. Revista Educación, 33(2), 15-24. https://www.redalyc.org/pdf/440/44012058002.pdf

Touron, J. (1984). Factores del rendimiento académico en la Universidad. Universidad de Navarra.

Universidad Nacional del Altiplano. (2019). Directiva Académica 2019.

Uturunco, Y. (2016). Factores que influyen en el rendimiento académico en universitarios de la Escuela de Enfermería [Universidad Nacional del Altiplano de Puno]. http://repositorio.unap.edu.pe/handle/UNAP/8644 
Vásquez, F. (2010). Estrategias de enseñanza: Investigaciones sobre didáctica en instituciones educativas de la ciudad de Pasto. Universidad de la Salle.

Vásquez, J. (2018). Hábitos de estudio y su relación con el rendimiento académico de los estudiantes de estomatología del I ciclo, 2015. Universidad Nacional de Educación «Enrique Guzman y Valle».

Vicuña, L. (1999). Inventario de hábitos de estudio CASM - 85. CEDEIS.

\section{Conflicto de intereses / Competing interests:}

Los autores declaran que no incurren en conflictos de intereses.

\section{Rol de los autores / Authors Roles:}

Wendy Soto: conceptualización, curación de datos, análisis formal, adquisición de fondos, investigación, metodología, administración del proyecto, recursos, software, supervisión, validación, visualización, escritura - preparación del borrador original, escritura - revisar \& amp; edición.

Nelly Rocha: conceptualización, investigación, metodología, administración del proyecto, recursos, software, supervisión, validación, visualización, escritura - preparación del borrador original, escritura - revisar \& amp; edición.

\section{Fuentes de financiamiento / Funding:}

Los autores declaran que no recibieron un fondo específico para esta investigación.

\section{Aspectos éticos / legales; Ethics / legals:}

Los autores declaran no haber incurrido en aspectos antiéticos, ni haber omitido aspectos legales en la realización de la investigación. 